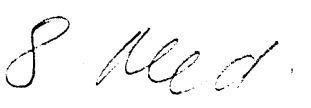

\title{
The Scandinavian Journal of
}

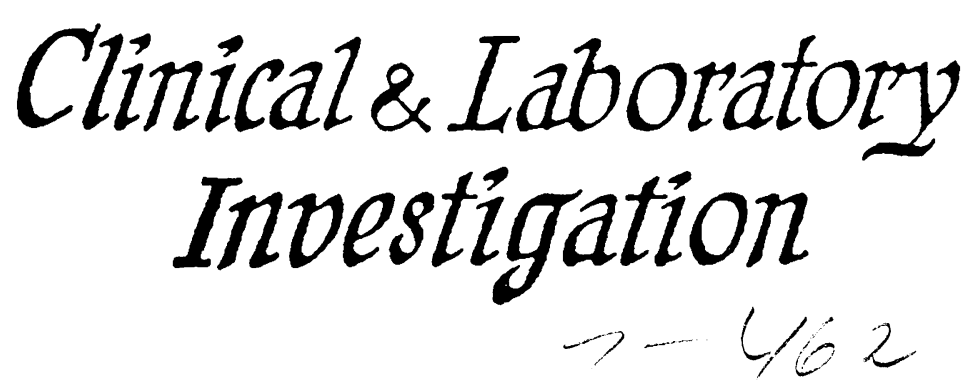

\author{
EDITED BY \\ THE SCANDINAVIAN SOCIETY \\ FOR CLINICAL CHEMISTRY \\ AND CLINICAL PHYSIOLOGY
}

\author{
MANAGING EDITOR \\ O. Stokke
}

\section{EDITORS}

J. Hartiala

J. H. Henriksen

E. Hultman

M. Hørder

K. Liewendahl
K. O. Pedersen

I. Romslo

K. Rootwelt

U-H. Stenman

EDITORIAL SECRETARIES

K. Bjøro

T. A. Hagve

Scand. J. Clin. Lab. Invest. - Vol. 50 - 1990 
Published for

Medisinsk Fysiologisk Forenings Forlag, Oslo by Blackwell Scientific Publications

Osney Mead, Oxford OX2 OEL

25 John Street, London WC1N 2BL

23 Ainslie Place, Edinburgh EH3 6AJ

3 Cambridge Center, Suite 208, Cambridge, MA 02142,

USA

54 University Street, Carlton, Victoria 3053, Australia

(C) 1990 Medisinsk Fysiologisk Forenings Forlag, Institute of Clinical Biochemistry, Rikshospitalet, N-0027 Oslo 1.

Printed and bound in Great Britain

by the Devonshire Press, Torquay
Authorization to photocopy for internal or personal use or the internal or personal use of specific clients is granted by Medisinsk Fysiologisk Forenings Forlag for libraries and other users registered with the Copyright Clearance Center (CCC) Transactional Reporting Service, provided that the base fec of $\$ 05.00$ per copy is paid directly to the CCC. 27 Congress Street, Salem, MA 01970, USA. This consent does not extend to other kinds of copying, such as copying for general distribution, for advertising or promotional purposes, for creating new collective works or for resale. Special requests should be addressed to the Editor. 0036-5513/90\$05.00.

Universilàts-

Binligrtie3k

München 


\section{Original Articles}

Sandwich enzyme-immunoassay of human urinary trypsin inhibitor (urinastatin) and urinastatin-like immunoreactive substance in mouse urine: T. Shikimi, S. Suzuki, M. Takahashi \& H. Kaneto

Functional capacity of neutrophil granulocytes in deep-sea divers: $H$. B. Benestad,

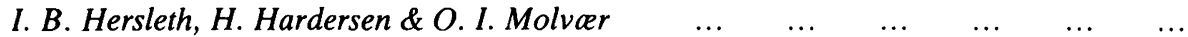

Pyrophosphate-loaded hepatocytes show increased iron uptake from transferrin: $T$. Nilson \& I. Romslo

Effects of exercise on glomerular passage of macromolecules in patients with diabetic nephropathy and in healthy subjects: I. Ala-Houhala

Release of immunoreactive canine leukocyte elastase normally and in endotoxin and pancreatitic shock: L. Axelsson, M. Bergenfeldt, P. Björk, R. Olsson \& K. Ohlsson $\ldots$

Release of lipoprotein lipase and hepatic lipase activities. Effects of heparin and a low molecular weight heparin fragment: E. Persson \& P. Nilsson-Ehle $\quad \ldots \quad \ldots \quad \ldots$

Monitoring of renal allografts by Doppler ultrasound: precision and reference values: $\begin{array}{llllllllll}K \text {. Rasmussen } \& \text { E. Pedersen } & \ldots & \ldots & \ldots & \ldots & \ldots & \ldots & \ldots & \ldots\end{array}$

Doppler ultrasound in the diagnosis of renal allograft rejection and in monitoring the effect

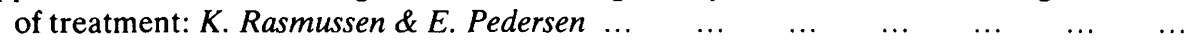

A method for determination of normal or abnormal blood volume in patients subjected to radionuclide cardiography: $O$. Carlsen \& $P$. Bruun $\ldots \begin{array}{lllllll}\ldots & \ldots & \ldots & \ldots & \ldots\end{array}$

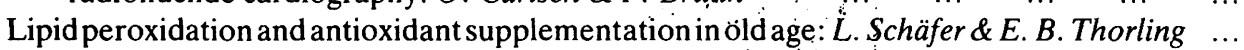
Serum ferritin and iron status in a population of 'healthy' 85 -year-old individuals: $N$. Milman, J. Ingerslev \& N. Graudal

The amino terminal portion of cerebrospinal fluid cystatin $\mathrm{C}$ in hereditary cystatin $\mathrm{C}$ amyloid angiopathy is not truncated: direct sequence analysis from agarose gel electropherograms:

I. Olafsson, G. Gudmundsson, M. Abrahamson, O. Jensson \& A. Grubb

Guidelines for description of immunoassay kits: A. Aakvag, E. Leskinen, G. Lindstedt,

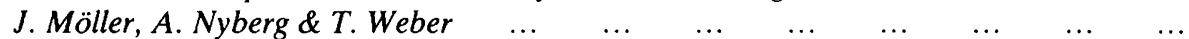

Recommendation for collection of venous blood from children, with special reference to production reference values: B. Lindblad, T. Alström, A. Bo Hansen, R. Gräsbeck, H. Hertz, C. Holmberg, E. Leskinen, P. J. Moe, A. P. W. Nyberg, V. Nänto, H. E. Solberg, L. Viinikka, P. D. Wimberley \& P. Winkel

\section{Letter to the Editor}

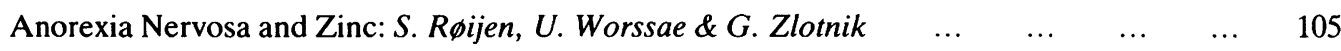
$\begin{array}{lllllllllllll}\text { List of Referees for } 1989 & \ldots & \ldots & \ldots & \ldots & \ldots & \ldots & \ldots & \ldots & \ldots & \ldots & 107\end{array}$ Erratum

\section{Original Articles}

Vitamin D metabolities-relation to age, menopause and endometriosis: D. Hartwell, $\begin{array}{llllll}P \text {. } R \phi d b r o, S \text {. } B \text {. Jensen, } K \text {. Thomsen } \& C \text {. Christiansen } & \ldots & \ldots & \ldots & \ldots & \ldots\end{array}$

Effect of acute vascular fluid volume expansion on erythrocyte sodium transport in essential
hypertension: $H$. Herlitz, O.K. Andersson, O. Jonsson, M. Wysocki, B. Persson \& M. Aurell

In vivo subcutaneous adipose tissue glucose kinetics after glucose ingestion in obesity and fasting: E. Hagström, P. Arner, P. Engfeldt, S. Rossner \& J. Bolinder 
No effect of bicarbonate-induced alkalosis on urea synthesis in normal man: K. Bjerrum,

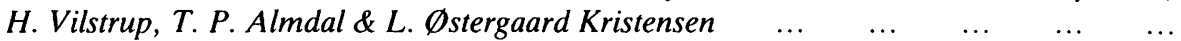

A sensitive method for measuring ATP-formation in rat muscle mitochondria: $R$. Wibom, A. Lundin \& E. Hultman

The effect of cobalt on mitochondrial ATP-production in the rat myocardium and skeletal muscle: N. Clyne, R. Wibom, N. Havu, E. Hultman, L-E. Lins, S. K. Pehrsson, B. Persson \& J. Rydström

Glomerular filtration rate estimated by X-ray fluorescence technique in children: comparison between the plasma disappearance of ${ }^{99} \mathrm{Tc}^{\mathrm{m}}$-DPTA and iohexol after urography:

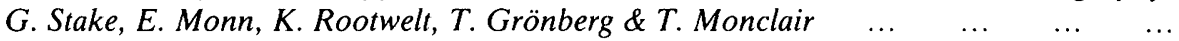

$\beta$-Adrenoceptor responsiveness in intact leukocytes from adults and newborn infants: effect of phosphodiesterase inhibitors: $N$. Foged, H. Bertelsen \& $T$. Johansen $\ldots \quad \ldots \quad \ldots$

Changes in the functional state of the erythrocyte membrane: significance for red cell filterability and blood viscosity: $H$. Larsson, S. U. Persson \& P. Hedner

Thromboplastin activity of blood monocytes after total hip replacement: $\emptyset$. P. Nygaard,

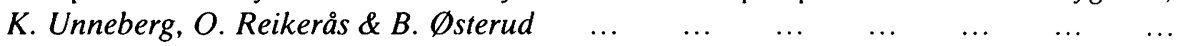

Epidermal growth factor and haptocorrin in nasal secretion: P. Tønnesen, L. Thim \& E. Nex $\emptyset$

Different behaviour of the $N$-terminal and $C$-terminal fragment of proatrial natriuretic factor in plasma of healthy subjects as well as of patients with cirrhosis: A. L. Gerbes, A. M. Vollmar, G. Thibault, R. M. Arendt, M. Cantin \& G. Paumgartner $\quad \ldots \quad \ldots$

Local sympathetic reflex control of blood flow in human skin following topical corticosteroid treatment: L. J. Petersen \& J. K. Kristensen

The effect of Acipimox on triacyglycerol metabolism in rat: A. Al-Shurbaji, L. Berglund \& I. Björkhem

Analysis of the relation between lithium clearance and sodium clearance in rats: $K$. Thomsen \& D. G. Shirley

\section{Technical Notes}

High- and low-affinity antibodies-some observations in relation to polyethylene glycol concentration in immunoturbidimetric assays: $K$. Larsson

Problems with the use of whole blood as a sample material in novel direct glucose analysers: $K$. Savolainen, A. Viitala, E. Puhakainen \& M. Väisänen

General Scandinavian recommendations on quality control and quality assurance in clinical chemistry

Comments on General Scandinavian recommendations on quality control and quality assurance in clinical chemistry

No. 3

\section{Review of a Scandinavian Thesis}

Proopiomelanocortin: $\alpha$-amidated and related peptides: $M$. Fenger $\quad \ldots \quad \ldots \quad \ldots \quad \ldots \quad \ldots$

\section{Original Articles}

The use of knowledge-based information systems for interpreting specialized clinical chemistry analyses - experience from erythrocyte enzymes and metabolites: F. Wiener,

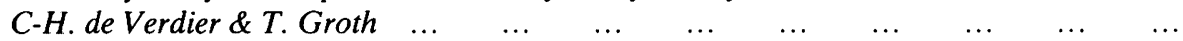

Reliability of the Reflotron in the determination of cholesterol: R. Selmer, O. P. Foss \& $\begin{array}{llllllllllll}P . & \text { G. Lund-Larsen } & \ldots & \ldots & \ldots & \ldots & \ldots & \ldots & \ldots & \ldots & \ldots & \ldots\end{array}$ 
Total body potassium, skeletal muscle potassium and magnesium in patients with Bartter's

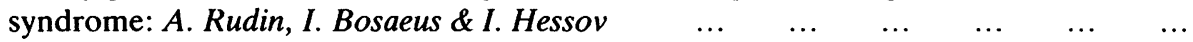

Determination of glomerular filtration rate using iohexol clearance and capillary sampling: E. Krutzén, S-E. Back \& P. Nilsson-Ehle

Diagnostic efficiency of lactate dehydrogenase isoenzymes in serum after acute myocardial

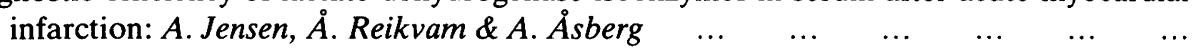

Reversibility of the effects on local circulation of high lipid concentrations in blood: J. Bülow, J. Madsen \& L. Højgaard

Blood cell filtrability: reference values and clinical applications: A. Belboul, N. Al-Khaja,

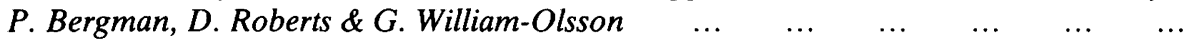

Increased urinary haemoglobin in diabetics with microalbuminuria-measured by an ELISA: $A$. Schmitz

Effect of retinoids and $1,25(\mathrm{OH})_{2}$ vitamin $\mathrm{D}_{3}$ bound to their plasma transport proteins on growth and differentiation of HL-60 cells: $M$. Botilsrud, I. Holmberg, K-O. Wathne,

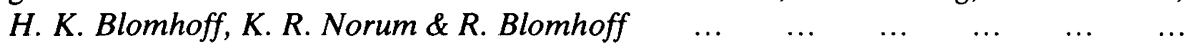

Solid-phase double-antibody radio-immunoassay for atrial natriuretic factor: $P$. H. Rasmussen, M. D Nielsen \& J. Giese

Transcapillary forces in muscle compartments of lower limbs with deep venous thrombosis: E. Seem \& E. Stranden

Transcapillary filtration in lower limbs with deep venous thrombosis: the role of the capillary filtration coefficient: $E$. Steem \& E. Stranden

Decreased concentrations of deoxycholic acid in serum of uraemic patients with diarrhoea: $\begin{array}{lllllll}P \text {. Stenvinkel, P. Bárány, I. Björkhem \& K. Einarsson } & \ldots & \ldots & \ldots & \ldots & \ldots\end{array}$ Isoelectric focusing of urinary metallothionein: E. Felley-Bosco, P. E. Hunziker \& K. Savolainen

Plasma HMW fibrinogen in patients with ischaemic heart disease: $\quad$ S. Munkvaad, W. Nieuwenhuizen \& J. Jespersen

No. 4

\section{Review of a Scandinavian Thesis}

Development of gangrene during sleep: $R$. Jelnes

\section{Original Articles}

Interactions between alkylglycerols and human neutrophil granulocytes: J. Palmblad, J. Samuelsson, \& J. Brohult

Atrial natriuretic factor, angiotensin II, aldosterone, arginine vasopressin and urinary prostaglandin $E_{2}$ excretion in hyperfiltrating unilaterally nephrectomized humans: S. S. Sørensen, B. Jespersen \& E. B. Pedersen $\quad \begin{array}{llllllll} & \ldots & \ldots & \ldots & \ldots & \ldots & \ldots\end{array}$

Lack of effect of dietary potassium on renal lithium clearance in man: Hla-Yee-Yee, D. G. Shirley, D. R. J. Singer, N. D. Markandu, M. G. Buckley, A L. Sugden, M. A. Miller \& G. A. MacGregor

Hormonal influence on calcitonin gene-related peptide in man: effects of sex difference and contraceptive pills: $S$. Valdemarsson, L. Edvinsson, P. Hedner \& R. Ekman $\quad \ldots \quad \ldots$

Comparative study of assays detecting complement activation. Complement-split product C3d (rocket immuno-electrophoresis) and C3d neodeterminants (ELISA):

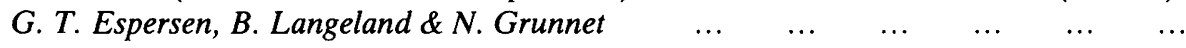
Increased erythrocyte magnesium content in essential hypertension: $S$. E. Kjeldsen, $O$. M. Sejersted, P. Frederichsen, P. Leren \& I. K. Eide 
Plasma cholinesterase activity in a healthy population group with no occupational exposure to known cholinesterase inhibitors: relative influence of some factors related to normal inter- and intra-individual variations: $A$. Brock \& $V$. Brock

Reproducibility of cumulative work, heart rate and blood pressure response during stepwise versus continuous load increment during a maximal bicycle ergometer test: $S$. Michelsen

Growth hormone $(\mathrm{GH})$ therapy in GH-deficient patients, the plasma Factor VIIIvon Willebrand factor complex, and capillary fragility. A double-blind, placebocontrolled crossover study: J. O. L. Jørgensen, S. A. Pedersen, J. Ingerslev, J. Møller, N. E. Skakkebok \& J. S. Christiansen

The production of tumour necrosis factor, tissue thromboplastin, lactoferrin and cathepsin $\mathrm{C}$ during lipopolysaccharide stimulation in whole blood: T. J. Gutteberg, B. Østerud,

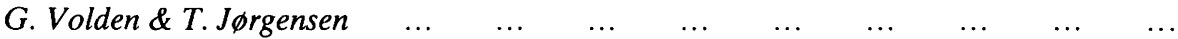

Primary platelet activation in recent-onset type 1 diabetes mellitus: A. M. Vicari \& A. Macagni

Molecular distribution of elastase between its two main inhibitors: direct quantitation of elastase- $\alpha_{2}$-macroglobulin complex with a novel ELISA technique: $E$. O. Adeyemi \& H. J. F. Hodgson

Validity and interpretation of blood lead levels: a study of Danish school children: T. Lyngbye,

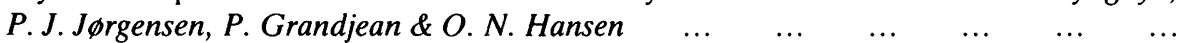

Xenon tissue/blood partition coefficient for pig urinary bladder: K. K. Nielsen, J. Bülow, S. L. Nielsen, J. Nordling \& B. Kromann-Andersen

\section{Expert Opinion}

Haemoglobin oxygen saturation and related quantities: definitions, symbols and clinical use: P. D. Wimberley, O. Siggaard-Andersen, N. Fogh-Andersen, W. G. Zijlstra \& J. W. Severinghaus

\section{Letter to the Editor}

Evidence of both decreased nuclear receptor-binding of $T_{3}$ and inhibited thyroid hormonestimulated glucose-uptake in cells from a euthyroid patient with hyperthyroxinaemia: J. Kvetny, L. E. Matzen \& M. Brøns

No. 5

\section{Review of a Scandinavian Thesis}

Clinical evaluation of cathodic trypsin-like immunoreactivity in pancreatic diseases in adults: J. Møller-Petersen

\section{Original Articles}

Pregnancy zone protein: a re-evaluation of serum levels in healthy women and in women suffering from breast cancer or trophoblastic disease: C. M. Petersen, P. H. Jensen,

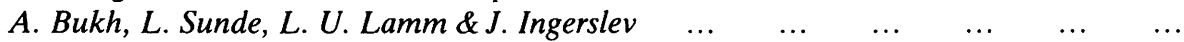

HPLC separation and quantification of bilirubin and its glucoronide conjugates in faeces and intestinal contents of germ-free rats: H. Saxerholt, V. Skar \& T. Midtvedt $\quad \ldots \quad \ldots$

A review of ${ }^{23} \mathrm{Na}$ nuclear magnetic resonance spectroscopy for the in vitro study of cellular sodium metabolism: H. Nissen, J. P. Jacobsen \& M. Hørder $\quad \begin{array}{lllll}\ldots & \ldots & \ldots & \ldots\end{array}$

Levels of neutrophil elastase and cathepsin B activities, and cystatins in human sputum: relationship to inflammation: D. J. Buttle, D. Burnett \& M. Abrahamson 
Bone mass measured by photon absorptiometry: comparison of forearm, heel, and spine: $M$. A. Hansen, B. J. Riis, K. Overgaard, C. Hassager \& C. Christiansen $\quad \ldots \quad \ldots$

The effect of exercise on urinary excretion of different-size proteins in patients with insulindependent diabetes mellitus: L. Groop, S. Stenman, P. H. Groop, A. Mäkipernaa \&

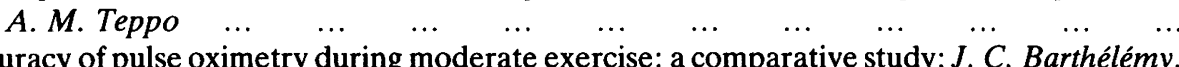

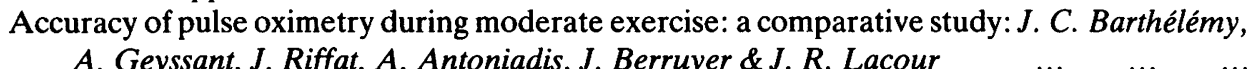

Analysis of purine nucleotides in muscle tissue by HPLC: J-P. Idström, B. Soussi, E. Wanag \& $A-C$. Bylund-Fellenius

Prevalence of anti-insulin antibodies and its relation to severe hypoglycaemia in insulintreated diabetic patients: $R$. Wredling, $P$. E. Lins \& U. Adamson

Serum ferritin, iron status and plasma ascorbic acid in 40- to 49-year-old males in the Faroe Islands: $N$. Milman, $H$. Thomsen \& B. Mathiassen

Age-associated decline of hepatic handling of cholephilic anions in humans is reverted by S-adenosylmethionine (SAMe): S. Gentile, M. Persico, C. Orlando, C. Le Grazie, C. Di Padova \& M. Coltorti

Reference intervals for immunoglobulins $\operatorname{IgA}$, IgG and $\operatorname{IgM}$ in serum in adults and in children aged 6 months to 14 years: K. Irjala, P. Koskinen, A. Icen \& T. Palosuo

Enhanced fractional sodium reabsorption in the ischaemic kidney revisited with lithium as a probe: N. E. Bruun, M. Rehling, P. Sk $\phi t$ \& J. Giese

\section{No. 6}

\section{Original Articles}

Distribution of bronchoalveolar cells and fibronectin levels in bronchoalveolar lavage fluids from patients with lung disorders: B. Nakstad, N. P. Boye \& T. Lyberg ... $\quad \ldots \quad \ldots$

Tissue protection by verapamil in the calcium paradox: S. Rotevath, G. Greve,

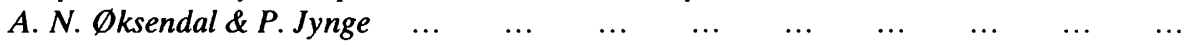

The intracellular distribution of cobalt in exposed and unexposed rat myocardium: $N$. Clyne, B. Persson, N. Havu, E. Hultman, L-E. Lins, S. K. Pehrsson, J. Rydström \& R. Wibom

Effect of enalapril on haemoglobin and serum erythopoietin in patients with chronic nephropathy: $A-L$. Kamper \& $O$. J. Nielsen

Determination of fibronectin and its degradation products in bronchoalveolar lavage fluids: E. Blaschke, A. Eklund \& R. Hernbrand

Excretion of carbonic anhydrase isozymes CA I and CA II in the urine of apparently healthy subjects and in patients with kidney disease: U. Backman, B. Danielsson \& P. J. Wistrand ...

Diurnal and monthly intra-individual variability of the concentration of lipids, lipoproteins and apoproteins: $A$. Wasenius, M. Stugaard, J. E. Otterstad \& D. Frøyshov $\quad \ldots \quad \ldots$

Automated cyanide-free method for haemoglobin determination on Technicon $\mathrm{H} \cdot 1$ : $\begin{array}{lllllllllllll}\text { L. Theodorsen } & \ldots & \ldots & \ldots & \ldots & \ldots & \ldots & \ldots & \ldots & \ldots & \ldots & \ldots\end{array}$

Plasma osteocalcin: biological variations and reference limits: P. Tarallo, J. Henny, B. Fournier \& G. Siest

Quantitative buffy coat analysis in haematological patients compared to standard laboratory methods: $T$. Lindhardt Pedersen, E. Kjarsgaard \& T. Plesner $\quad \begin{array}{llllll} & \ldots & \ldots & \ldots & \ldots\end{array}$

Ultrafiltration devices tested for use in a free thyroxine assay validated by comparison with equilibrium dialysis: $S$. Tikanoja

Correlations between psychological and physiological responses to acute flight phobia stress: O. Ekeberg, S. E. Kjeldsen, D. T. Greenwood \& E. Enger 
Compartmental analysis of short-lived platelet dynamics: S. Savolainen, M. T. Syrjälä,

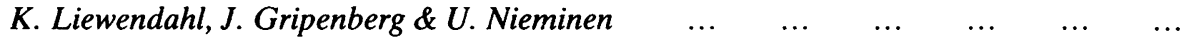

Adsorption of creatinine to Fuller's earth: J. Egense, C. J. W. Koch \& M. Willems $\ldots \quad \ldots$

Stanozolol and experimental atherosclerosis: atherosclerotic development and blood lipids during anabolic steroid therapy of New Zealand White rabbits: $M$. Fogelberg, I. Björkhem, U. Diczfalusy \& P. Henriksson

\section{No. 7}

\section{Original Articles}

The secretory erythropoietin response in patients with multiple myeloma and Waldenstrom's macroglobulinaemia: O. J. Nielsen, M. Brandt \& A. Drivsholm

Interleukin-2- and neopterin-induced neopterin release from peripheral blood mononuclear cells: M. Barak, D. Merzback \& N. Gruener

Analytical goal setting prior to selection of a method for glycated haemoglobin: $M$. Lytken Larsen, O. Blaabjerg, P. Hyltoft Petersen, H. Hansen \& M. Hørder

Determination of serum levels of unesterified lanosterol by isotope dilution-mass

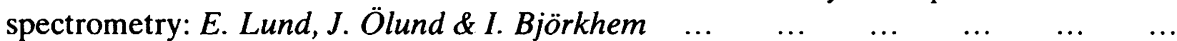

The elimination of secretory leukocyte protease inhibitor (SLPI) after intravenous injection in dog and man: $M$. Bergenfeldt, P. Bjork \& K. Ohlsson

Impairment by glycation of immunoglobulin G Fc fragment function: $R$. Dolhofer-Bliesener \& K. D. Gerbitz

New ergometric reference values for clinical exercise tests: $M$. Arstila, O. Impivaara \& J. $M a ̈ k i$

Plasma and renal clearance of iohexol-a study on the reproducibility of a method for the

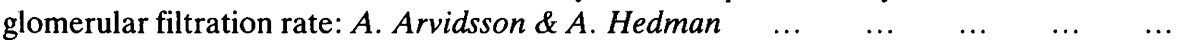

Elimination of ${ }^{14} \mathrm{C}$-glycated albumin from serum of rabbit: $A$. Kallner $\quad \ldots \quad \ldots \quad \ldots$

L1, a major granulocyte protein; isolation of high quantities of its subunits: H. B. Berntzen \& M. K. Fagerhol

Distribution of ethanol and water between plasma and whole blood; inter- and intraindividual variations after administration of ethanol by intravenous infusion: A. W. Jones, R. G. Hahn \& H. P. Stalberg

Lactate balance in perfused rat liver: effects of glucose concentration, flow and low $\mathrm{pH}$ on glucose to lactate flux: $L$. Sestoft \& M.O. Marshall

Gradual reduction of coronary perfusion pressure in cats: changes in transmural distribution of blood flow: S. Birkeland, E. Hexeberg, K. Matre, P. Kvitting, J. Westby, J. Lekven \& K. Grong

Plasma volume changes during hypoglycaemia: the effect of arterial blood sampling: J. Hilsted, F. Bendtsen, N. J. Christensen \& J. H. Henriksen $\quad \ldots \quad \ldots \quad \ldots \quad \ldots$

757

Different short-term effect of protein and carbohydrate intake on TSH, growth hormone $(\mathrm{GH})$, insulin, $C$-peptide, and glucagon in humans: L. E. Matzen, B. B. Andersen, B. G. Jensen, H. J. Gjessing, S. H. Sindrup \& J. Kvetny

No. 8

\section{Original Articles}

Observations on biochemical and haematological parameters in healthy people above 80 years of age: $B$. Lantz, A. Molander \& G. Nilsson

Renal haemodynamic and sympathetic responses to head-up tilt in essential hypertension:

A. Westheim, I. Os, S. E. Kjeldsen, E. Føntelien \& I. K. Eide 
Autonomic response to an experimental psychological stressor in healthy subjects: measurement of sympathetic, parasympathetic, and pituitary-adrenal parameters: test-retest reliability: L. S. Jørgensen, P. Christiansen, U. Raundahl, S. Østgaard, N. J. Christensen, M. Fenger \& Helga Flachs

The influence of induced hyperglycaemia on gastric emptying rate in healthy humans: E. Øster-Jorgensen, $S$. A. Pedersen \& M. L. Larsen $\quad \begin{array}{lllllll}\ldots & \ldots & \ldots & \ldots & \ldots\end{array}$

Plasma levels of the calcium-binding L1 leukocyte protein: standardization of blood collection and evaluation of reference intervals in healthy controls: $I$. Dale $\quad \ldots \quad \ldots$

Preanalytical handling of stored urine samples, and measurement of $\beta_{2}$-microglobulin, orosomucoid, albumin, transferrin and immunoglobulin $\mathrm{G}$ in urine by enzyme-linked immunosorbent assays (ELISA): E. Vittinghus

Diurnal rhythm in serum activity of wheat-germ lectin-precipitable alkaline phosphatase: temporal relationships with the diurnal rhythm of serum osteocalcin: $H$. K. Nielsen, K. Brixen \& L. Mosekilde

Identification and quantification of serum proteins secreted into the normal human jejunum: $V$. Andersen \& J. H. Hegnhøj

Vascular clearance by the reticuloendothelial system-measurements using two differentsized albumin colloids: S. B. Holmberg, E. Forssell-Aronsson, J. Gretarsdottir, L. Jacobsson, B. Rippe \& L. Hafström

Pyridoxine reduces cholesterol and low-density lipoprotein and increases antithrombin III activity in 80-year-old men with low plasma pyridoxal 5-phosphate: L. Brattström, L. Stavenow, H. Galvard, P. Nilsson-Ehle, E. Berntorp, P. Jerntorp, S. Elmstahl \& H. Pessah-Rasmussen

A modified cytotoxicity assay with high sensitivity: $U$. Keilholz, R. Dummer, H. Welters,

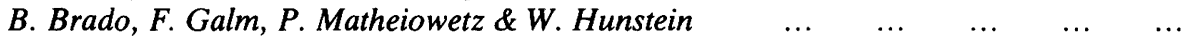

Performance of two agglutination techniques in the detection of thyroid antibodies and assessment of their clinical significance: E. Soppi, A-M. Haapala, S. Laine \& J. Salmi

Induced hypocalcaemia controlled by a citrate clamp technique, and the intact parathyroid hormone response obtained: P. Schwartz, H. A. Sørensen, I. Transbøl \& P. McNair ...

In vitro catabolism of histamine in the human stomach: H. Lönroth, G. Granerus, $\begin{array}{lllllllllll}\text { E. Rosengren \& L. Lundell } & \ldots & \ldots & \ldots & \ldots & \ldots & \ldots & \ldots & \ldots & \ldots\end{array}$

Immunoturbidimetric determination of pregnancy-specific $\beta_{1}$-glycoprotein (SP-1): $\begin{array}{llllllllll}U \text {. Turpeinen \& } U-H \text {. Stenman } & \ldots & \ldots & \ldots & \ldots & \ldots & \ldots & \ldots & \ldots\end{array}$

\section{Technical Note}

Evaluation of a simple method for the screening of microalbuminuria in diabetic patients: A. S. Al-Kassab

\section{Letter to the Editor}

Limits of lipoprotein $\mathrm{Lp}(\mathrm{a})$ detection by polyacrylamide gel electrophoresis in discontinuous

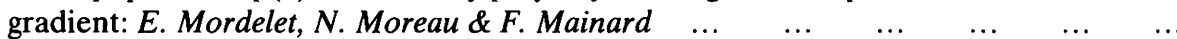





\section{Key-words}

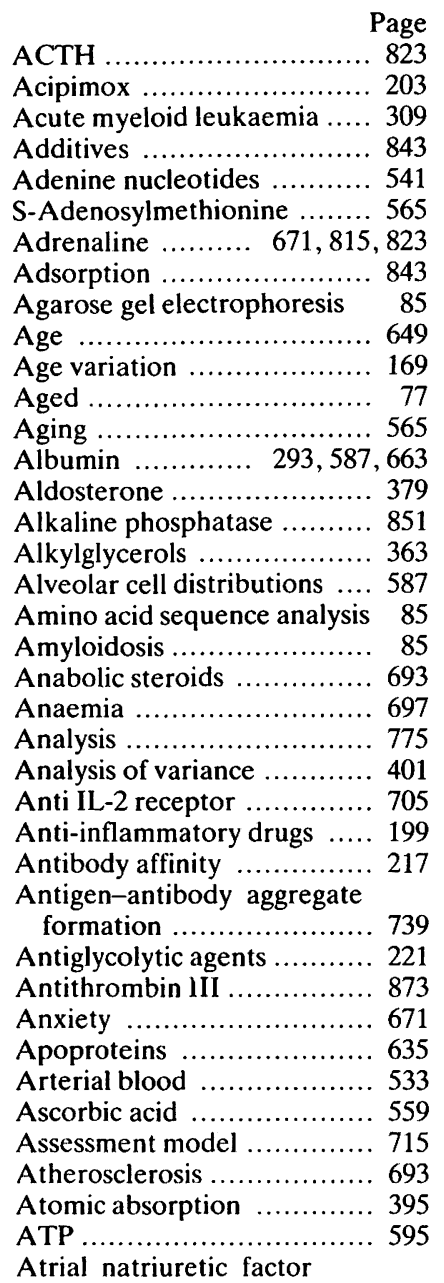

(ANF) …............. 195, 319

Atrial natriuretic peptide

(ANP) ........................ 379

Autoimmune ................ 885

Autoinduction .................. 705

Automated haemoglobin

determination ...............6 643

Autonomic response .......... 823

Autoregulation ................ 787

Barters syndrome ............. 275

Bicycle ergometry ............. 747

Bile acids ...................... 337

Bilirubin ....................... 565

Bilirubin conjugates ............ 487

Biochemistry ................. 807

Bioluminescence .............. 143

Blood cell counting ............. 657

Blood cell

deformability ......... 177, 299

Blood collection

99, 837

\begin{tabular}{|c|c|}
\hline Page & Contraceptives \\
\hline Blood flow ................ & $\begin{array}{l}\text { Contraceptives } \\
\text { Contrast media }\end{array}$ \\
\hline lood flow regulation. & Contrast media \\
\hline Blood gases $\ldots \ldots \ldots \ldots \ldots \ldots$ & Copper ................ \\
\hline d lead concentration & Coronary stenosis ... \\
\hline od lipids $\ldots \ldots \ldots \ldots \ldots \ldots \ldots . \ldots 6$ & Cortisol $\ldots \ldots \ldots \ldots \ldots$ \\
\hline d pressure $\ldots . .395,671,815$ & ${ }^{51} \mathrm{Cr}$-EDTA extraction \\
\hline d proteins ................... 857 & ${ }^{51} \mathrm{Cr}$-release . \\
\hline ood rheology ....... & Cystatin C .. \\
\hline y fluids & Cysteine proteinase inhibitor \\
\hline he isoenzyme $\ldots \ldots \ldots \ldots \ldots \ldots$ & Cytotoxicity $\ldots \ldots \ldots \ldots \ldots \ldots$ \\
\hline
\end{tabular}

Bone markers ................... 851

Bone mineral content ......... 517

Bone mineral density ......... 517

Bovine albumin ................ 843

Breast cancer .................. 479

Bronchiectasis ................... 509

Bronchoalveolar lavage ....... 619

Bronchoalveolar lavage

fluids ........................... 587

Butyrylcholinesterase .......... 401

C3d (RIE) ..................... 389

C3d neodeterminants

(ELISA) ....................... 389

cAMP …............... 169, 595

Cadmium ........................... 343

Calcitonin gene-related

peptide ......................... 385

Calcium ........................... 177

Calcium content ................ 595

Calf blood flow ................ 331

Calprotectin ........................ 769

Canine endotoxin shock ....... 35

Capillary blood sampling ..... 441

Capillary filtration

coefficient ................... 331

Carbonic anhydrase ........... 627

Catecholamines ......... 797, 815

Cathepsin C .................... 421

Cell injury ..................... 595

Cellular mediated immunity.. 705

Cerebral haemorrhage ....... 85

Chemiluminescence ........... 363

Children .......................... 99

Cholesterol ........................ 873

Cholesterol precursor .......... 723

Cholesterol synthesis .......... 723

Chronic nephropathy ...........611

Chylomicron remnants ........ 309

Circadian rhythm ............. 851

Cirrhosis ............................ 195

Clearance ........................... 865

Cobalamin ................... 187

Cobalamin-binding proteins 187

Cobalt .................... 153, 605

Colloid ....................... 865

Colloid osmotic pressure …... 326

Compartment models ......... 679

Complement activation

measurements ................. 389

Complement fixation ........... 739
Deconjugation ................. 487

Degradation ............. 619,857

5'-Deiodination ............... 801

Delivery from proximal

tubules ........................ 209

Deoxycholic acid ............... 337

Dextrans ......................... 27

Diabetes ............................ 739

Diabetes mellitus ............... 913

Diabetic nephropathy .... 27,305

Diarrhoea ….................. 337

Dietary sodium content ....... 209

Discriminant analysis ......... 285

Diseases ........................... 299

Distribution ................... 775

Diurnal variability .............. 635

Divided renal function ......... 579

Doppler velocity ............ 51,57

Dose-response curves ........ 217

Dot-blot ....................... 769

Dry-chemistry ..................... 263

Dual photon absorptiometry 517

Duodenal ulcer ................. 899

Duration of insulin treatment .................... 551

Elastase inhibitors ............. 35

Elastase- $\alpha_{2}$-macroglobulin complex .................... 433

Elastase- $\alpha_{1}$-protein inhibitor complex ...................... 433

ELISA …..................... 433

Elimination ...................... 775

Enalapril ........................... 611

Endometriosis ................... 115

Energy metabolism ............ 541

Environmental surveillance 441

Ephedrine ........................ 187

Erythrocyte magnesium ....... 395

Erythrocyte-zincprotoporphyrin ............. 441

Erythropoietin ........... 611,697

Essential hypertension ........ 395

Ethanol ........................ 775

Exercise ................ 27, 525, 533

Exercise test ................... 409

Factor VIII ..................... 417

Faeces ............................ 487
Epidemiology .................... 441 


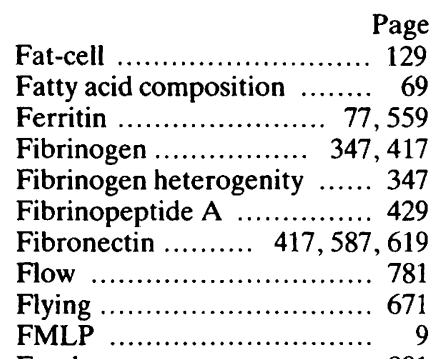

Food ......................... 801

Fractional lithium excretion.. 379

Free fatty acids ......... 203, 293

Free-water reabsorption ..... 371

Freezing ....................... 843

Fructosamine ............... 763

Gastric emptying ............ 831

Germ-free life ................ 487

Glomerular filtration rate

(GFR) .............. 371, 757

Glomerular permeability ...... 27

Glucocorticoids ............... 1

Glucose .................... 831

Glucose concentration ........ 781

Glucose dehydrogenase ...... 221

Glucose determination ........ 221

Glucose oxidase ............... 221

Glucuronidase, $\beta$............. 487

Glycated haemoglobin ....... 715

Glycolysis .................... 781

Glycolytic enzymes ........... 247

${ }^{3} \mathrm{H}$-thymidine release ........ 879

Haematocrit ................... 451

Haematology ................ 807

Haemodynamic parameters 409

Haemoglobin ... 77, 441, 559, 661

Haemoglobinometry .......... 643

HbA ........................ 763

Healthy individuals ........... 409

Healthy subject ............... 635

Healthy subjects .............. 649

Healthy volunteers ............ 757

Heparin ..................... 43

Hepatic lipase ................ 43

Hepatocytes ................... 19

Hereditary disorders .......... 247

High-performance liquid

chromatography (HPLC)... 281

Highly purified insulins ....... 551

Histamine .................... 187

Histamine catabolism ........ 899

HMG CoA reductase ......... 723

Human stomach ............... 899

Hydrogen peroxide

electrode ................... 221

Hypercalcuria ................ 627

Hyperoxia ................... 9

Hypertension .................. 123

Hypoglycaemia ................ 797

Hypolipidaemic drug ......... 203

Hypoxia oxgen monitoring ... 533
Idiopathic thrombocytopenic

$$
\text { purpura }
$$

IgG ...................... 679 679

Immobilized enzymes ......... 221

Immunoassay kits ............. 95

Immunoglobulin assay

calibration

573

$\begin{array}{lll}\text { Immunoturbidimetry ... } & 217,907\end{array}$

Imprecision .................... 715

Inaccuracy ................. 715

Indicator dilution method .... 63

Induced hypocalcaemia ...... 891

Insulin ...................... 797

Insulin dose ................... 551

Insulin-dependent diabetes

mellitus (IDDM) 27, 305, 525

Intact parathyroid hormome 891

Intercalated disc .............. 595

Interleukin-2 ................ 879

Interstinal fluid ............... 129

Interstitial fluid hydrostatic pressure ................... 331

Interstitial fluid pressure ..... 326

Intestinal contents ............ 487

Intestinal secretions .......... 857

Intracellular calcium concentration ............... 363

Iodination ...................... 319

Iodogen ..................... 319

Iohexol .................. 161, 757

Iron metabolism ......... 77, 559

Iron uptake ................. 19

Ischaemia ...................... 541

Ischaemic heart disease ...... 347

Isobutyl-methyl-xanthine ..... 169

Isoelectric focusing .......... 343

Isolated perfused rat heart .... 595

Jejunum

Kidney transplantation .... 51,57

Kinetics ...................... 763

Kinetics, plasma ............. 775

Knowledge-based information system

L1 protein ..................... 837

L1 subunits .................... 769

LAK cells ...................... 879

Lactate uptake ............... 781

Lactoferrin .................... 421

Lead exposure ............... 441

Letter to editor .......... 105, 461, 917

Leukocyte ............... 9, 729

Leukocyte elastase (canine).. 35

Likelihood ratio ................ 285

Lipid emulsion ................ 293

Lipopolysaccharide .......... 421

Lipoprotein lipase ........... 43

Lipoproteins .................. 635

Lithium clearance ............ 209

Lithium extraction ............. 579

Liver .......................... 565

Liver function ............... 137
Liver scintigraphy .............. 865

Lloyd's reagent ................ 687

Long-term quality

assessment ................... 247

Low molecular weight heparin 43

Low-density lipoproteins .... 873

Lung disorders ................ 587

Macroglobulin, $\alpha_{2} \ldots \ldots . \quad 35,433$ 479,509

Macrophage ............ 705, 865

Males ......................... 559

Mass fragmentography ....... 723

Membrane fluidity ............. 565

Menopause .................... 649

Menstrual cycle ................ 649

Mental arithmetic ............. 823

Metabolic control ............. 551

Metabolism ................... 801

Metabolism of human

erythrocytes .............. 247

Methacholine ................. 187

Microalbuminuria ............. 913

Microdialysis .................. 129

Microsomal .................... 885

Microspheres ................ 293

Mitochondrial

ATP-production

Monocyte tissue

thromboplastin ............ 421

Monocytes .................. 183

Monthly variability ............ 635

Morphometric parameters .... 649

Mouse urine ................... 1

Multiple myeloma ............ 697

Multiple regression analysis 401

Myeloperoxidase ............. 509

Myocardial blood flow ........ 787

Myocardial ischaemia ........ 787

Nephelometry ................ 217

Nephrectomy ................ 371

Neuropeptides ................. 385

Neutrophil degranulation ..... 509

Nicotinic acid .................. 565

Nitrogen metabolism ......... 137

Non-insulin dependent diabetes

(NIDD) .................. 305

Noradrenaline ..... 671,815,823

Normal subjects ............... 115

Normal values ................. 837

Nutrition ....................... 801

Occult haematuria ........... 305

Oestrogens ..................... 385

$1,23(\mathrm{OH}) 2 \mathrm{D}$................. 115

$24,25(\mathrm{OH}) 2 \mathrm{D} \ldots \ldots \ldots \ldots \ldots . . .115$

$25(\mathrm{OH}) \mathrm{D}$.................... 115

Osteoblastic activity ............. 851

Osteoporosis ............. 115, 517

Ouabain ...................... 177

Oxidative phosphorylation ... 143

Oxygen dissociation curve..... 455 
Oxygen radicals ............... 541

Oxyhaemoglobin fraction ..... 455

$\mathrm{pH}$ 137,781

Pancreatitis 35

Peripheral blood mononuclear cells ......................... 705

Phenyl methyl sulphonyl fluoride (PMSF) ............ 433

Phobia .......................... 671

Phosphatidate phosphohydrolase .......... 203

Physical capacity ............... 747

Physical fitness ................ 747

Pig ...................... 293, 451

Pituitary hormones ........... 801

Plasma bone Gla protein (BGP) …................... 649

Plasma concentration ......... 837

Plasma renin activity (PRA).. 379

Plasma viscosity ................ 177

Plasma volume ................. 797

Plasminogen ...................... 417

Plasminogen activator ........ 417

Platelet dynamics ............... 679

Platelet-associated IgG ....... 679

Platelets ........................ 429

PMA ........................... 9

Polymorphonuclear granulocytes ............... 363

Positional vasomotor activity ....................... 199

Posture ....................... 195

Potassium intake .............. 379

Pregnancy ..................... 907

Pregnancy zone protein (PZP) ....................... 479

Pregnancy-associated $\alpha_{2}$-glycoprotein .............. 479

Preparative isoelectric focusing ....................... 769

Progesterone .................. 385

Promyelocyte ................. 309

Protease ......................... 729

Protease inhibitor ............... 729

Protein A binding .............. 739

Proteinase inhibitor, $\alpha_{1} . .35,433$

Proteinuria .......... 27, 525, 627

Proximal renal tubules ........ 579

Pseudocholinesterase .......... 401

Puberty ........................ 649

Pulsatility index ........... 51,57

Pulse oximetry ................... 533

Purified L1 protein ............ 769

Pyridoxal 5-phosphate ........ 873

Pyridoxine ...................... 873

Pyrophosphate ................ 19

Quantitative buffy coat (QBC) analysis ........................... 657

Rabbits .......................... 693

Radio-immunoassay ......... 319, 663,907
Page

Rat myocardium ........ 153, 605

Ratios .......................... 775

Receiver operating

characteristic .................. 285

Red blood cells ................. 63

Red cell membrane ............. 177

Referees for 1989 , list of ..... 107

Reference limits ............... 649

Reference method ............... 687

Reference values ............ 99, 299

Regional perfusion ............. 293

Regulation ..................... 801

Relaxation studies ............. 497

Renal blood flow .............. 815

Renal clearance ................ 161

Renal function ....... 281, 343, 371

Renal resistance ............ 51,57

Renal tubular acidosis ......... 627

Renin-angiotensin system .... 123

Renovascular hypertension $\quad 579$

Repeatability .................. 823

Repeated-measures analysis of

variance ...................... 401

Respiratory burst ............ 9

Reticuloendothelial system (RES)

865

Retinoid acid .................. 309

Retinol ............................ 309

Reverse-phase HPLC .......... 319

Sampling studies

Sandwich enzyme-

immunoassay ................. 1

Sarcoidosis ........................ 619

Scandinavian ...................... 99

Screening ................. 907, 913

Selenium ......................... 69

Senescence ...................... 807

Serial assessment ............. 409

Serodia ........................... 885

Serotonin ........................ 187

Severe hypoglycaemia ......... 551

Shift reagents .................... 497

Single-injection clearance .... 281

Single photon absortiometry 517

Skeletal muscle .......... 153,541

Skeletal muscle magnesium... 275

Skeletal muscle potassium .... 275

Small intestine (human) ....... 857

Smoking ......................... 619

Sodium ........................... 371

Sodium clearance ............. 209

Sodium flux ...................... 497

${ }^{23}$ Sodium-NMR theory ......... 497

Sodium transport ............... 123

Sodium-potassium pump ..... 177

Soleus muscle .................. 143

Specificity ….................... 715

Stanzolol ............................ 693

Strain-gauge

plethysmography ............ 331

Stress ........................... 823

Subcellular fractions ............ 605
Page

Superoxide anion formation.. 363

Surgery ....................... 183

Sympathetic tone .............6 671

TSH ........................... 807

Technetium-99m $\left({ }^{(9)} \mathrm{Tc}^{\mathrm{m}}\right) \ldots . .63$

Technicon H-1 ................. 643

Thawing ........................... 843

Theophylline .................. 169

Thiobarbituric (TBA) test ... 69

Thromboplastin activity ....... 183

Thrombosis ...................... 183

Thromboxane B2 .............. 429

Thymune ....................... 885

Thyroglobulin ................. 885

Thyroid hormone ............... 663

Thyroiditis ...................... 885

Thyroxine ...................... 801

Tissue composition ............... 451

Tissue/blood partition

coefficient ..................... 451

Topical .............................. 199

Total body potassium ......... 275

Total cholesterol ................. 263

Total gastrectomy ............... 899

Total hip replacement ......... 183

Total plasma clearance ........ 161

Transcapillary filtration ....... 326

Transferability .................. 573

Transferrin ............... 77, 559

Thromboglobulin, $\beta$........... 429

Triacylglycerol synthesis ...... 203

Triacylglycerol secretion ...... 203

Triiodothyronine .............. 801

Trophoblastic disease ......... 479

Tumour necrosis factor ........ 421

Tween-20 ....................... 843

Two-dimensional

electrophoresis ............. 769

Type 1 diabetes ................. 551

Type 1 diabetes mellitus ...... 429

Ultrastructure .................. 595

Uraemia ......................... 337

Urea synthesis ................. 137

Urinary albumin

concentration ................ 305

Urinary metalothionein ........ 343

Urinary trypsin inhibitor ...... 1

Urinastatin .................... 1

Urinastatin-like substance .... 1

Urography ......................... 161

Vagus ........................... 831

Vascular resistance ............... 417

Veno-arteriolar reflex .......... 199

Venous blood ................... 99

Viability ........................... 497

Visibility .......................... 497

Vitamin A ..................... 309

Vitamin B12 ................. 807

Vitamin D-binding protein

(DBP) 


\begin{tabular}{|c|c|}
\hline Page & Page \\
\hline Vitamin $E \ldots \ldots \ldots \ldots \ldots \ldots \ldots \ldots \ldots \ldots \ldots \ldots$ & Wet-chemistry .............. \\
\hline Volume load ................... 123 & Whole blood ................. 775 \\
\hline von Willebrand factor ......... 417 & $\begin{array}{l}\text { Wick-in-needle } \\
\quad \text { technique } \ldots \ldots \ldots \ldots \ldots \quad 326,331\end{array}$ \\
\hline $\begin{array}{l}\text { Waldenström's } \\
\text { macroglobulinaemia ....... } 697\end{array}$ & $\begin{array}{lr}\text { Women } \ldots \ldots \ldots \ldots \ldots \ldots \ldots \ldots \ldots & 69 \\
\text { Work capacity evaluation ..... } & 747\end{array}$ \\
\hline
\end{tabular}

\section{Authors}

Aakvaag, A. ................ 95

Abrahamson, M. ........ 85, 509

Adamson, U. ................. 551

Adeyemi, E.O. ............... 433

Al-Kassab, A.S. ............. 913

Al-Khaja, N. ................. 299

Al-Shurbaji, A. .............. 203

Ala-Houhala, I. ............. 27

Almdal, T.P. ................. 137

Alström, T. .................. 99

Andersen, B.B. ............ 801

Andersen, V. ................. 857

Andersson, O.K. ............. 123

Antoniadis, A. .............. 533

Arendt, R.M. ............... 195

Arner, P. .................... 129

Arstila, M. .................... 747

Arvidsson, A. ................ 757

Åsberg, A. ................... 285

Aurell, M. .................. 123

Axelsson, L. ............... 35

Back, S-E. ................... 281

Backman, U. ................ 627

Barak, M. ................. 705

Barany, P. ................... 337

Barthélémy, J.C. ............ 533

Belboul, A. .................. 299

Bendtsen, F. .................. 797

Benestad, H.B. ............... 9

Bergenfeldt, M. ......... 35, 729

Berglund, L. .................. 203

Bergman, P. ................. 299

Berntorp, E. ................ 873

Berntzen, H.B. ............... 769

Berruyer, J. .................. 533

Bertelsen, H. .............. 169

Birkeland, S. ................ 787

Bjerrum, K. ................ 137

Björk, P. ............... 35, 729

Björkhem, I. ... 203, 337, 693, 723

Blaabjerg, O. ................ 715

Blaschke, E. ................ 619

Blomhoff, H.K. ............ 309

Blomhoff, R. ................ 309

Bo Hansen, A. ............... 99

Bolinder, J. .................. 129

Bosaeus, I. .................. 275

Botilsrud, M. ................ 309

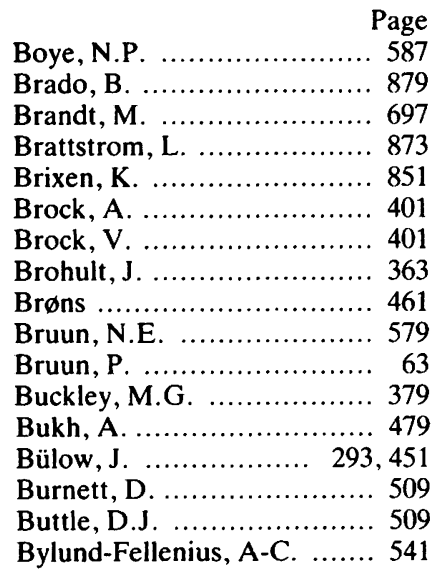

Cantin, M. .................. 195

Carlsen, O. .................... 63

Christensen, N.J. .............. 797

Christiansen, C. ......... 115, 517

Christiansen, J.S. ............. 417

Christiansen, P. ............... 823

Christiansen, N.J. ............ 823

Clyne, N. .............. 156, 605

Coltorti, M. .................. 565

Dale, I. ...................... 837

Danielsson, B. ................ 627

De Verdier, C-H. ............. 247

Di Padova, C. ................. 565

Diczfalusy, U. ................. 693

Dolhofer-Bliesener, R. ....... 739

Drivsholm, A. ................ 697

Dummer, R. ................. 879

Edvinsson, L. ................ 385

Egense, J. ................... 687

Eide, I.K. ............... 395, 815

Einarsson, K. ................ 337

Ekeberg, O. ................... 671

Eklund, A. .................. 619

Ekman, R. .................. 385

Elmstahl, S. ................. 873

Enger, E. ................... 671

Engfeldt, P. .................. 129

Espersen, G.T ............... 389

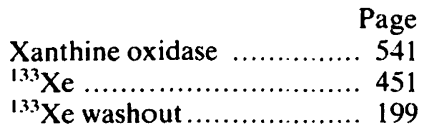

Zinc 343

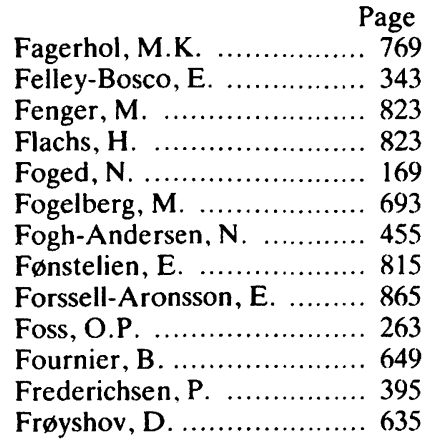

Galm, F. ................... 879

Galvard, H. ............... 873

Gentile, S. .................. 565

Gerbes, A.L. ................. 195

Gerbitz, K.D. ............... 739

Geyssant, A. ............... 533

Giese, J. ............... 319,579

Grandjean, P. ............... 441

Granerus, G. ................ 899

Gräsbeck, R. ............... 99

Graudal, N. .................. 77

Greenwood, D.T. ............ 671

Gretarsdottir, J. .............. 865

Greve, G. ................... 595

Gripenberg, J. ............... 679

Grönberg, T. ............... 161

Grong, K. ................... 787

Groop, L. .................... 525

Groop, P.H. ............... 525

Groth, T. ................... 247

Grubb, A. .................... 85

Gruener, N. ................. 705

Grunnet, N. ................. 389

Gudmundsson, G. ........... 85

Gutteberg, T.J. ............. 421

Haapala, A-M. ............... 885

Hafström, L. ................ 865

Hagström, E. ............... 129

Hahn, R.G. .................. 775

Hansen, H. ................... 715

Hansen, M.A. ................ 517

Hansen, O.N. ............... 441

Hardersen, H. ................. 9

Hartwell, D. ................ 115 


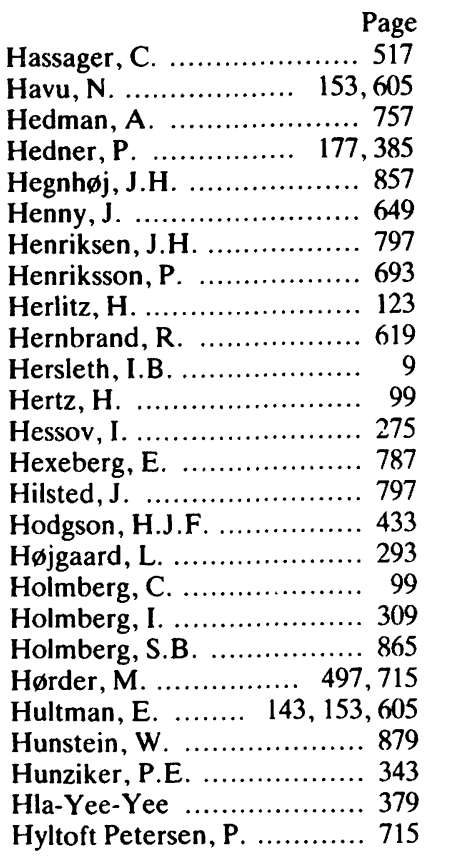

Icen, A. ....................... 573

Idström, J-P. ................ 541

Impivaara, O. ............... 747

Ingerslev, J. ......... 77, 417, 479

Irjala, K. .................... 573

Jacobsen, J.P. ................. 497

Jacobsson, L. ...................... 865

Jensen, A.E. .................... 285

Jensen, B.G. . ................ 801

Jensen, P.H. ................. 479

Jensen, S. B. .................. 115

Jensson, O.................. 85

Jerntorp, P. .................. 873

Jespersen, J. ...................... 347

Jespersen, K. ................. 371

Johansen, T. . ................. 169

Jones, A.W. . ..................... 775

Jonsson, O. ................. 123

Jørgensen, J.O.L. ............ 417

Jorgensen, L.S. . ............. 823

Jorgensen, P.J. ................ 441

Jørgensen, T. ................ 421

Jynge, P. ......................... 595

Kallner, A. ..................... 763

Kamper, A-L. ................ 611

Kaneto, H. ................... 1

Keilholz, U. ........................ 879

Kjærsgaard, E. .............. 657

Kjeldsen, S.E. ...... 395, 671, 815

Koch, C.J.W. .................. 687

Koskinen, P. ................... 573

Kristensen, J.K. .............. 199

Kromann-Andersen, B. ....... 451

Krutzén, E. .................... 281
Page

Kvetny, J. ............. 461, 801

Kvitting, $\mathbf{P}$.

787

Lacour, J.R.

533

Laine, S. ..................... 885

Lamm, L.U. .................... 479

Langeland, B. ................ 389

Lantz, B. .................... 807

Larsen, M.L. ................ 831

Larsson, H. ................. 177

Larsson, K. ................... 217

Le Grazie, C. ................... 565

Lekven, J. ........................ 787

Leren, P. ....................... 395

Leskinen, E. . ............ 95, 99

Liewendahl, K. ................. 679

Lindblad, B. ................... 99

Lindhardt Pedersen, T. ...... 657

Lindstedt, G. ................ 95

Lins, L-E. .......... 153, 605, 551

Lönroth, H. .................... 899

Lund, E. ........................... 723

Lund-Larsen, P.G. ............ 263

Lundell, L. .................... 899

Lundin, A. ................... 143

Lyberg, T. ......................... 587

Lyngbye, T. .................. 441

Lytken Larsen, M ............ 715

MacGregor, G.A. ............ 379

Macagni, A. ................... 429

Madsen, J. .................... 293

Mäki, J. ........................... 747

Mäkipernaa, A. .............. 525

Markandu, N.D. ............. 379

Marshall, M.O. ................. 781

Matheiowetz, P. ............. 879

Mathiassen, B. .............. 559

Matre, K. ...................... 787

Matzen, L.E. ........... 461, 801

McNair, P. ..................... 891

Merzbach, D. ................. 705

Michelsen, S. ................... 409

Midtvedt, T. ................ 487

Miller, M.A. -............... 379

Milman, N. ................. 77, 559

Moe, P.J. ....................... 99

Molander, L. ................ 807

Möller, J. .................... 95, 417

Møller-Petersen, J. ............. 463

Molvær, O.I. .................. 9

Monclair, T. .................. 161

Monn, E. .......................... 161

Mosekilde, L. ................. 851

Munkvad, S. ................. 347

Nakstad, B. .................... 587

Nänto, V. ..................... 99

Nexø, E. .......................... 187

Nielsen, H.K. ............... 851

Nielsen, K.K. .................... 451

Nielsen, M.D. ................... 319

Nielsen, O.J. ............. 611, 697
Page

Nielsen, S.L. .................. 451

Nieminen, U. .................... 679

Nieuwenhuizen, W. .......... 347

Nilsen, T. . . .................. 19

Nilsson, G. .................... 807

Nilsson-Ehle, P. ..... 43, 281, 873

Nissen, H. ..................... 497

Nordling, J. ................... 451

Norum, K.R. . ............... 309

Nyberg, A. ...................... 95

Nyberg, A.P.W. .............. 99

Nygaard, $\varnothing . P . \ldots \ldots \ldots \ldots \ldots 183$

Ohlsson, K. ............... 35, 729

Øksendal, A.N. ............... 595

Olafsson, 1. .................... 85

Olsson, R. ....................... 35

Ölund, J. ..................... 723

Orlando, C. ................... 565

Os, I. ............................. 815

Oster-Jorgensen, E. ............ 831

Østergaard-Kristensen, L. .... 137

Østerud, B. ............... 183, 421

Østgaard, S. .................... 823

Otterstad, J.E. ................. 635

Overgaard, K. .................. 517

Palmblad, J. ................... 363

Palosuo, T. ..................... 573

Paumgartner, G. .............. 195

Pedersen, E. ............... 51, 57

Pedersen, E. B. ................ 371

Pedersen, S.A. ......... 471, 831

Pehrsson, S.K. .......... 153, 605

Persico, M. ................. 565

Persson, B. ........ 123, 153, 605

Persson, E. ....................... 43

Persson, S.U. ................... 177

Pessah-Rasmussen, H. ....... 873

Petersen, L.J. ................... 199

Peterson, C.M. ................... 479

Plesner, T. ................... 657

Puhakainen, E. ................ 221

Rasmussen, K. ........... 51, 57

Rasmussen, P.H. .............. 319

Raundahl, U. ................... 823

Rehling, M. ................... 579

Reikrås, $\mathrm{O} \ldots \ldots \ldots \ldots \ldots \ldots \ldots \ldots$

Reikvam, Å. ...................... 285

Riffat, J. . ........................ 533

Riis, B.J. ....................... 517

Rippe, B. .................... 865

Roberts, D. ..................... 299

Rødbro, ....................... 115

Røijen, S. .................... 105

Romslo, I. ................... 19

Rootwelt, K. .................... 161

Rosengren, E. ................. 899

Rossner, S. ................... 129

Rotevatn, S. .................... 595

Rudin, A. ...................... 275

Rydström, J. ............ 153, 605 


\begin{tabular}{|c|c|c|}
\hline Page & Page & Page \\
\hline Salmi, J..... & Stalberg, H.P. ............... 775 & Vicari, A.M. ...... \\
\hline Samuelsson, J. & Stavenow, L. $\ldots \ldots \ldots \ldots \ldots . .873$ & Viinikka, L. .. \\
\hline Savolainen, $\mathrm{H}$. & Stenman, S. .... & Viitala, A........ \\
\hline Savolainen, K. . & Stenman, U-H. & Vilstrup, H. .. \\
\hline Savolainen, S. & Stenvinkel, P. . & Vittinghus, $\mathrm{E}$. \\
\hline Saxerholt, $\mathrm{H}$. & Stranden, E. .... & Volden, G. ... \\
\hline Schäfer, L. ... & Stugaard, $\mathrm{M} . \ldots$. & Vollmar, A.M. . \\
\hline Schmitz, A. & Sugden, A.L. ..................... 379 & \\
\hline Schwarz, P. .. & Sunde, L. ...................... 479 & \\
\hline 331 & Suzuki, S. .................. 1 & Wanag, E. ... \\
\hline 325 & Syrjälä, M.T. & Wasenius, $\mathrm{A}$. \\
\hline ted, O.M. & & Wathne, K-O. \\
\hline 263 & Takahashi, M. .................. & Weber, T. ...... \\
\hline Sestoft, L. & Tarallo, P. ....................... & Weiner, F. \\
\hline ghaus, J.W. & Teppo, A.M. .. & 879 \\
\hline Shikimi, T. .................. 1 & Theodorsen, L. & Westby, J. \\
\hline Shirley, D.G. ........... 209,379 & Thibault, G. .... & Westheim, A. \\
\hline Siest, G. ...................... 649 & Thim, L. ...... & .. $143,153,605$ \\
\hline Siggard-Andersen, $O \ldots \ldots$ & Thomsen, $\mathrm{H}$. & Willems, M. . \\
\hline Sindrup, S.H. ............... 801 & Thomsen, K..... & William-Olsson, G. \\
\hline Singer, D.R.J. ................ 379 & Thorling, E.B. . & Wimberley, P.D. ... \\
\hline Skakkebæk, N.E. ............ 417 & Tikanoja, S. .. & Winkel, $\mathrm{P} . \ldots$. \\
\hline 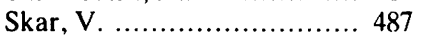 & Tønnesen, P. ................ 187 & Wistrand, P.J. \\
\hline Skøtt, P. ..................... 579 & Transbol, I. .................. 891 & \\
\hline Solberg, H.E. ...................... 99 & 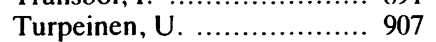 & Wredling, $\mathrm{R}$. \\
\hline 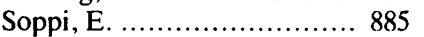 & & Wysocki, M. .. \\
\hline Sørensen, H.A. .............. 891 & Unneberg, K. .................. 183 & \\
\hline Sørensen, S.S. ................ 371 & & \\
\hline ................. 541 & Väisänen, M. ...... & Zijlstra, W.G .. \\
\hline 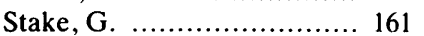 & Valdemarsson, S. ............ 385 & Zlotnik, G. .................. 105 \\
\hline
\end{tabular}




\title{
Different behaviour of the $N$-terminal and $C$-terminal fragment of proatrial natriuretic factor in plasma of healthy subjects as well as of patients with cirrhosis
}

\author{
A. L. GERBES, A. M. VOLLMAR, ${ }^{*}$ G. THIBAULT ${ }^{\dagger}$ \\ R. M. ARENDT, M. CANTIN ${ }^{\dagger} \&$ G. PAUMGARTNER
}

Departments of Medicine II and I, Klinikum Grosshadern, *Institute of Pharmacology, Toxicology and Pharmacy, University of Munich, Munich, FRG and ${ }^{\dagger}$ Clinical Research Institute of Montreal, Montreal, Quebec, Canada

Gerbes AL, Vollmar AM, Thibault G, Arendt RM, Cantin M, Paumgartner G. Different behaviour of the $N$-terminal and $C$-terminal fragment of proatrial natriuretic factor in plasma of healthy subjects as well as of patients with cirrhosis. Scand J Clin Lab Invest 1990; 50: 195-198.

$N$-terminal (atrial natriuretic factor (ANF) 1-98) and $C$-terminal (ANF 99-126) fragments of proatrial natriuretic factor (NTA and CTA, respectively) were determined in plasma of healthy subjects adopting different postures and in patients with cirrhosis. Seven healthy subjects were investigated while seated and $30 \mathrm{~min}$ after assuming a horizontal position. NTA plasma concentrations increased in subjects in the horizontal position (from $734 \pm 250$ (SE) $\mathrm{fmol} / \mathrm{ml}$ to $902 \pm 227$ $\mathrm{fmol} / \mathrm{ml} ; \mathrm{p}<0.05$ ). In contrast, CTA plasma concentrations remained unchanged $(9.2 \pm 1.3 \mathrm{fmol} / \mathrm{ml}$ vs $8.9 \pm 1.6 \mathrm{fmol} / \mathrm{ml})$. In 10 patients with cirrhosis of the liver, NTA concentrations were markedly $(p<0.001)$ elevated compared to 11 healthy subjects $(2334 \pm 291 \mathrm{fmol} / \mathrm{ml}$ vs $743 \pm 155 \mathrm{fmol} / \mathrm{ml})$. However, there was no difference of CTA plasma levels between cirrhotic patients and healthy subjects $(8.7 \pm 1.3 \mathrm{fmol} / \mathrm{ml}$ vs $8.2 \pm 0.9 \mathrm{fmol} / \mathrm{ml})$. These data demonstrate changes of the plasma concentration of the $\mathrm{N}$-terminal fragment of proatrial natriuretic factor by posture and in liver disease, in contrast to unchanged levels of the $C$-terminal fragment.

Key words: atrial natriuretic factor (ANF); cirrhosis; posture

A. L. Gerbes MD, Medizinische Klinik II, Klinikum Grosshadern, University of Munich, Marchioninistrasse 15, D-8000 München 70, Federal Republic of Germany

Proatrial natriuretic factor (atrial natriuretic factor (ANF) 1-126) is stored in atrial granules, released and cleaved upon appropriate stimulation; the $C$-terminal fragment, CTA (ANF 99-126) has been identified as biologically active circulating peptide (for review see $[1,2]$ ).
Recently, presence of the $N$-terminal fragment NTA (ANF 1-98) has been demonstrated in plasma of rat and man [3, 4] and there is increasing evidence for cosecretion of NTA with CTA [3-5]. Plasma concentrations of NTA have been found several-fold higher compared to 
respective CTA concentrations [3-6], probably owing to a longer half-life of NTA [3]. Therefore, an increase of pro-ANF release may be more sensitively detected by determining the $\mathrm{N}$-terminal than the $\mathrm{C}$-terminal fragment in plasma. To test this hypothesis, in the present study NTA and CTA plasma concentrations were determined upon a change of posture, which may be regarded as a subtle stimulus of proANF release.

More pronounced elevations of NTA than of CTA have been observed in plasma of patients with chronic renal failure, suggesting a role of the kidney in eliminating NTA from the circulation [4]. The possible role of the liver, the other major organ for clearance of endogenous compounds, in the elimination of NTA has not yet been addressed. Therefore, in the present study NTA and CTA plasma concentrations were determined in patients with chronic liver disease.

\section{MATERIALS AND METHODS}

\section{Subjects}

Eleven healthy subjects, aged 25 to 65 (mean $37 \pm 4$ ) years and 10 patients with cirrhosis of the liver (histologically confirmed, mostly in advanced stage with ascites), aged 47 to 75 (mean 58 \pm 3 ) years, were investigated (sitting position) after their informed consent had been obtained. The subjects showed no evidence of cardiovascular, renal or pulmonary disease. They were on a regular diet, containing approximately $150 \mathrm{meq}$ sodium/day.

\section{Change of posture}

Seven healthy subjects, aged 20 to 65 (mean $35 \pm 6$ ) years were investigated. Blood samples were taken after they had been sitting comfortably on a chair for $30 \mathrm{~min}$. Thereafter, they assumed a horizontal position, lying on a bed in supine position. Thirty min after this change of posture another blood sample was taken. Heart rate and blood pressure were recorded in both positions.

\section{Determination of ANF}

CTA was determined by radio-immunoassay (RIA) with antibody 'Toni III' (no evidence of cross-reactivity with NTA), as described in detail previously [7]. The RIA procedure for
NTA with antiserum 'GT 23' (no cross-reactivity with CTA detectable) has been reported in detail [3]. Immunoreactivity has been identified as CTA and NTA, respectively, by highperformance liquid chromatography (HPLC) techniques [5, 7]. Plasma renin activity was determined as described by Wernze et al. [8].

\section{Statistical evaluation}

Differences of CTA and NTA plasma concentrations, of plasma renin activity, heart rate and mean arterial pressure, induced by the change of posture were calculated by paired t-test. CTA and NTA plasma levels of healthy subjects and controls were compared by unpaired t-test. A value of $\mathrm{p}<0.05$ was considered significant. Data are presented as mean and standard error.

\section{RES ULTS}

\section{Change of posture}

NTA concentrations in seven healthy subjects were about a hundred-fold higher $(p<0.001)$ than the corresponding CTA concentrations (Table I). The change of posture to the supine position did not affect CTA levels, whereas NTA concentrations significantly increased $(\mathrm{p}<0.05)$. Mean arterial pressure did not change, the heart rate decreased $(p<0.01)$ and plasma renin activity tended to decreasc $(p<0.08)$.

\section{ANF in patients with cirrhosis}

In sitting position, CTA concentrations in 10 patients with cirrhosis were not different from those in 11 healthy subjects $(8.7 \pm 1.3 \mathrm{fmol} / \mathrm{ml}$ is

TABIE I. Effects of change of posture in seven healthy subjects

\begin{tabular}{lccc}
\hline & Sitting & Supine & $p$ \\
\hline $\begin{array}{c}C \text {-terminal ANF } \\
(\text { fmol/ml) }\end{array}$ & $8.9 \pm 1.6$ & $9.2 \pm 1.3$ & -- \\
$\begin{array}{c}N \text {-terminal ANF } \\
(\text { fmol/ml) }\end{array}$ & $734 \pm 250$ & $902 \pm 227$ & $<0.05$ \\
$\begin{array}{c}\text { Plasma renin activity } \\
(\mathrm{ng} \mathrm{AI} / \mathrm{ml} / \mathrm{h})\end{array}$ & $2.6 \pm 0.5$ & $1.6 \pm 0.3$ & $<0.08$ \\
$\begin{array}{c}\text { Heart rate } \\
(\mathrm{min}-1)\end{array}$ & $69.7 \pm 3.6$ & $61.1 \pm 2.1$ & $<0.01$ \\
$\begin{array}{c}\text { Mean arterial } \\
\text { pressure }(\mathrm{mmHg})\end{array}$ & $93.1 \pm 3.6$ & $95.7 \pm 6.1$ & - \\
\hline
\end{tabular}




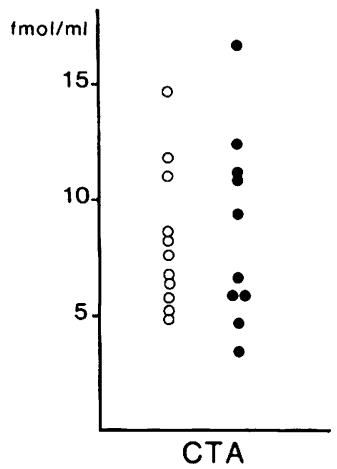

o healthy subjects

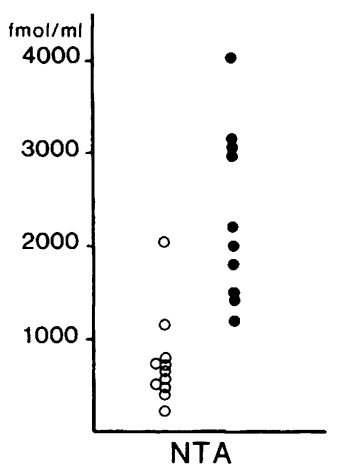

- patients with cirrhosis
FIG. 1. Plasma concentrations of $C$-terminal (CTA) and $N$-terminal (NTA) fragments of proatrial natriuretic factor in 11 healthy subjects $(O)$ and 10 patients with cirrhosis $(\mathbf{O})$.

$8.2 \pm 0.9 \mathrm{fmol} / \mathrm{ml}$ ). However, mean plasma concentrations of NTA were markedly $(p<0.001)$ higher in cirrhosis than in controls $(2334 \pm 291 \mathrm{fmol} / \mathrm{ml}$ vs $743 \pm 155 \mathrm{fmol} / \mathrm{ml}$ ) (Fig. 1). Renal function, as estimated by serum creatinine concentrations was no different in patients with cirrhosis when compared with healthy subjects $(1.10 \pm 0.03 \mathrm{mg} / 100 \mathrm{ml}$ vs $1.03 \pm 0.05$ $\mathrm{mg} / 100 \mathrm{ml}$ ).

\section{I S C US S I O N}

The influence of posture on plasma concentrations of $C$-terminal ANF has been the subject of some controversy, with some authors observing differences $[9,10]$ while others did not $[11,12]$. In the present study, the concentration of the $C$ terminal fragment was not influenced by the change from sitting to supine position. However, the change of posture initiated a significant increase of $\mathrm{N}$-terminal fragment concentrations. The decrease of heart rate as well as that of plasma renin activity in the supine position suggests a subtle increase of central vascular filling, which may be regarded as a stimulus of pro-ANF release. Thus, the likely augmentation of ANF release by the change of posture was reflected by the change of NTA concentrations, and not reflected by CTA determinations.
Whereas many authors agree that CTA plasma levels are unchanged in cirrhosis [13], some report elevated levels (for review see [14]). In the present study, we found no difference in the plasma concentrations of CTA in patients with cirrhosis or healthy subjects. However, concentrations of the $\mathrm{N}$-terminal fragment were elevated in cirrhosis by about threefold. This finding might be compatible with the contention of increased release of ANF in cirrhosis [15], but only in the presence of an enhanced clearance of $C$-terminal ANF.

Since clearance of CTA seems unaltered in cirrhosis [16], this hypothesis is rather unlikely. Another explanation for increased NTA at normal CTA concentrations could be an impaired elimination of the $N$-terminal fragment in cirrhosis. Renal elimination of NTA has been suggested, based on the observation of more marked elevations of NTA than of CTA in patients with chronic renal failure [4]. In the cirrhotic patients of this study, normal serum creatinine levels make significant renal impairment rather unlikely. However, in cirrhosis, renal blood flow can be reduced at unchanged serum creatinine concentrations; thus, the possibility cannot be excluded that the observed increase of NTA in cirrhosis could be partly caused by decreased renal elimination. Furthermore, influence of age cannot be excluded since patients and controls were not age-matched. However, the striking elevations of NTA observed in patients with cirrhosis suggest that the liver plays a role in the elimination of NTA from the circulation.

\section{A C K N OWLEDGMENTS}

We thank Professor H. Wernze, University of Würzburg for the determinations of plasma renin activity; M. Bauch, G. Hach and U. Rüberg for technical assistance; and F. Höpker and R. Witthaut for preparation of the manuscript. This study was supported by the Deutsche forschungsgeineinschaft (Ge 57612-1).

\section{R E FER E N C E S}

1 Cantin M, Genest J. The heart as an endocrine gland. Sci Am 1986; 254: 76-81.

2 Needleman P. Atriopeptin: biochemical pharmacology. Federation Proc 1986; 45: 2096-100. 
3 Thibault G, Murthy $\mathrm{KH}$, Gutkowska J et al. $\mathrm{NH}_{2}-$ terminal fragment of rat pro-atrial natriuretic factor in the circulation: identification, radioimmunoassay and half-life. Peptides 1988; 9: 47-53.

4 Sundsfjord JA, Thibault G, Larochelle P, Cantin M. Identification and plasma concentrations of the $\mathrm{N}$-terminal fragment of proatrial natriuretic factor in man. J Clin Endocrinol Metabol 1988; 66: 605-10.

5 Gerbes AL, Vollmar AM. Water immersion increases the concentration of the immunoreactive $\mathrm{N}$-terminal fragment of proatrial natriuretic factor in human plasma. Biochem Biophys Res Comm 1988; 156: 228-32.

6 Gauquelin G, Thibault G, Cantin M, Schiffrin EL, Garcia R. Glomerular atrial natriuretic factor receptors during rehydration: plasma $\mathrm{NH}_{2}$ - and COOH-terminal levels. Am J Physiol 1988; 255: F621-5.

7 Arendt RM, Gerbes AL, Ritter D, Stangl E. Differential processing of plasma atrial natriuretic factor in cardiovascular disease. In: Brenner BM, Laragh JH, eds. Biologically Active Atrial Peptides, Vol I. New York: Raven Press, 1987: 544-7.

8 Wernze H, Spech HJ, Müller G, Schweinsfurth H, Schöner $\mathrm{H}$, Brügel $\mathrm{H}$. The renin-angiotensinaldosterone system (RAAS) in chronic liver disease. In: Addison GM et al., eds. Aldosterone Antagonists in Clinical Medicine. Amsterdam: Excerpta Medica 1978: 394-404.

9 Larose P, Meloche S, du Souich P, De Léan A, Ong H. Radioimmunoassay of atrial natriuretic factor: human plasma levels. Biochem Biophys Res Comm 1985; 130: 553-8.
10 Ogihara T, Shima J, Hara H, Kumuhara Y, Kangawa $\mathrm{K}$, Matsuo $\mathrm{H}$. Changes in human plasma atrial natriuretic polypeptide concentration in normal subjects during passive leg raising and whole-body tilting. Clin Sci 1986; 71: 147-50.

11 Hodsman GP, Phillips PA, Ogawa K, Johnston CI. Atrial natriuretic factor in normal man: effects of tilt, posture, exercise and hemorrhage. J Hypertension 1986; 4 (Suppl. 6): 503-5.

12 Jüppner H, Brabant G, Kapteina U, Kirschner M, Klein $\mathrm{H}$, Hesch RD. Direct radioimmunoassay for human atrial natriuretic peptide (hANP) and its clinical evaluation. Biochem Biophys Res Comm 1986; 139: 1215-23.

13 Gerbes AL, Wernze H, Arendt RM, Riedel A, Sauerbruch T, Paumgartner G. Atrial natriuretic factor and renin-aldosterone in volume regulation of patients with cirrhosis. Hepatology 1989; 9: 417-422.

14 Gerbes AL, Arendt RM, Paumgartner G. Editorial review. Atrial natriuretic factor - possible implications in liver disease. J Hepatol 1987; 5: $123-32$.

15 Gines $\mathrm{P}$, Jiménez W, Arroyo $\mathrm{V}$ et al. Atrial natriuretic factor in cirrhosis with ascites: plasma levels, cardiac release and splanchnic extraction. Hepatology 1988; 8: 636-42.

16 Henriksen JH, Schütten HJ, Bendtsen F, Warberg J. Circulating atrial natriuretic peptide (ANP) and central blood volume (CBV) in cirrhosis. Liver 1986; 6: 361-8.

Received 1 July 1989

Accepted 20 September 1989 